
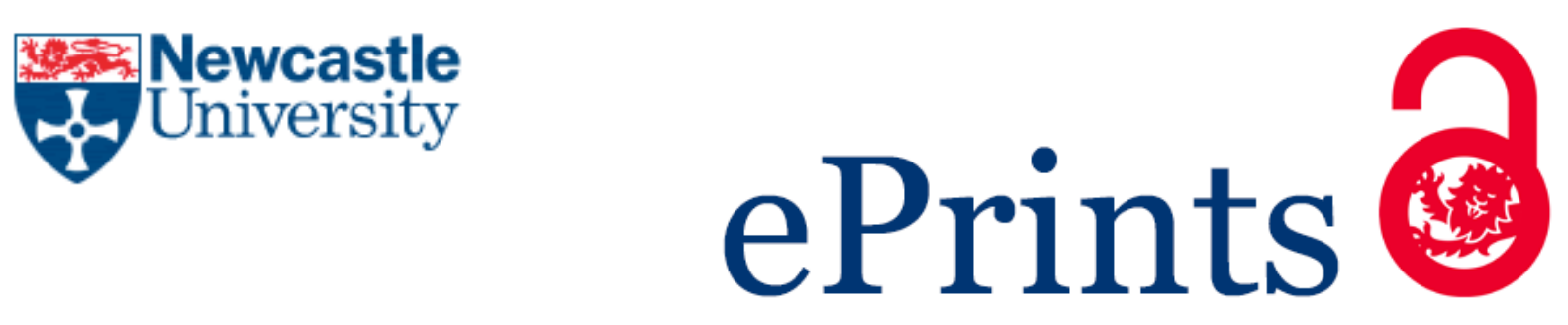

Rostami N, Shields RC, Yassin SA, Hawkins AR, Bowen L, Luo TL, Rickard AH, Holliday R, Preshaw PM, Jakubovics NS.

A Critical Role for Extracellular DNA in Dental Plaque Formation. Journal of Dental Research (2016)

DOI: $\underline{\text { http://dx.doi.org/10.1177/0022034516675849 }}$

\title{
Copyright:
}

This is the authors' accepted manuscript of an article that has been published in its final definitive form by Sage Publications Ltd, 2016.

DOI link to article:

http://dx.doi.org/10.1177/0022034516675849

Date deposited:

$02 / 11 / 2016$

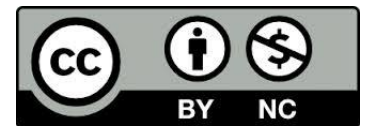

This work is licensed under a Creative Commons Attribution-NonCommercial 3.0 Unported License 


\section{A Critical Role for Extracellular DNA in Dental Plaque Formation}

Nadia Rostami ${ }^{1}$, Robert C. Shields ${ }^{1}$, Sufian A.Yassin ${ }^{1}$, Alastair R. Hawkins ${ }^{2}$, Leon Bowen ${ }^{3}$, Ting

L. Luo ${ }^{4}$, Alexander H. Rickard ${ }^{4}$, Richard Holliday ${ }^{1}$, Philip M. Preshaw ${ }^{1}$ and Nicholas S.

Jakubovics $^{1 *}$

1. School of Dental Sciences, Newcastle University, Newcastle upon Tyne, UK; 2. Institute for Cell and Molecular Biosciences, Newcastle University, Newcastle upon Tyne, UK; 3.

Department of Physics, Durham University and 4. Department of Epidemiology, University of Michigan, Ann Arbor, MI, USA

\section{Address for correspondence:}

Nicholas S. Jakubovics

Centre for Oral Health Research

School of Dental Sciences

Newcastle University

Framlington Place

Newcastle upon Tyne NE 2 4BW

UK

Tel: +1912086796

Fax: +191 2088807

Email: nick.jakubovics@newcastle.ac.uk

\section{Running title:}

Extracellular DNA in dental plaque

\section{Keywords:}

Biofilm(s), Dental implant(s), Microbial ecology, Microbiology, Microscopy, Plaque/Plaque biofilms 


\section{ABSTRACT}

Extracellular DNA (eDNA) has been identified in the matrix of many different mono-

3 species biofilms in vitro, including some of those produced by oral bacteria. In many cases, eDNA

4 stabilizes the structure of mono-species biofilms. Here, we aimed to determine whether eDNA is

5 an important component of natural mixed-species oral biofilms such as plaque on natural teeth or

6 dental implants. To visualize eDNA in oral biofilms, approaches for fluorescently staining eDNA

7 with either anti-DNA antibodies or an ultrasensitive cell-impermeant dye, YOYO-1, were first

8 developed using E. faecalis, an organism that has previously been shown to produce extensive

9 eDNA structures within biofilms. Oral biofilms were modelled as in vitro 'microcosms' on glass

10 coverslips inoculated with the natural microbial population of human saliva and cultured statically

11 in artificial saliva medium. Using antibodies and YOYO-1, eDNA was found to be distributed

12 throughout microcosm biofilms, and was particularly abundant in the immediate vicinity of cells.

13 Similar arrangements of eDNA were detected in biofilms on crowns and overdenture abutments

14 of dental implants that had been recovered from patients during the restorative phase of treatment,

15 and in subgingival dental plaque of periodontitis patients, indicating that eDNA is a common

16 component of natural oral biofilms. In model oral biofilms, treatment with a DNA-degrading

17 enzyme, NucB from Bacillus licheniformis, strongly inhibited the accumulation of biofilms. The

18 bacterial species diversity was significantly reduced by treatment with NucB and particularly

19 strong reductions were observed in the abundance of anaerobic, proteolytic bacteria such as

20 Peptostreptococcus, Porphyromonas and Prevotella. Pre-formed biofilms were not significantly

21 reduced by NucB treatment, indicating that eDNA is more important or more exposed during the

22 early stages of biofilm formation. Overall, these data demonstrate that dental plaque eDNA is

23 potentially an important target for oral biofilm control. 


\section{INTRODUCTION}

25 Dental caries, periodontitis and peri-implantitis are caused by the accumulation of

26 microbial biofilms (plaque) on tooth or implant surfaces. Any individual harbors approximately

27 100-300 different species/phylotypes from a pool of around 700 common oral bacteria (Chen et

28 al. 2010; Zhang et al. 2015). These complex communities are held together by a matrix of

29 extracellular polymers, including polysaccharides, lipids, proteins and nucleic acids. Attempts

30 have been made to control biofilms by targeting extracellular polysaccharides using enzymes such

31 as glucanases or dispersin B (Otsuka et al. 2015; Ragunath et al. 2016). However, even relatively

32 simple mixed-species biofilms can contain many different polysaccharides and the complexity of

33 polysaccharide linkages limits the efficacy of individual enzymes for degrading biofilm matrices.

34 Consequently, research has focused on other structurally relevant extracellular biofilm molecules

35 that may be more susceptible to degradation and, in particular, extracellular DNA (Okshevsky et

36 al. 2014).

37 Extracellular DNA (eDNA) stabilizes many different laboratory-grown mono-species

38 biofilms (Jakubovics et al. 2013; Okshevsky and Meyer 2015). Biofilm matrix eDNA can

39 potentially be targeted by bacterial DNases such as NucB from Bacillus licheniformis, which can

40 be produced cost-effectively in microbial expression systems (Rajarajan et al. 2012). However, at

41 present little is known about the role of eDNA in mixed-species biofilms and even the detection

42 of eDNA in complex natural biofilm communities has proven challenging. Using fluorescent dyes

43 and DNase treatment, eDNA has been detected in activated sludge flocs (Dominiak et al. 2011).

44 There is also circumstantial evidence that eDNA is present in dental plaque. For example,

45 photochemical cross-linking of extracellular DNA with propidium monoazide reduced the

46 concentration of PCR-amplifiable $S$. mutans DNA in dental plaque by approximately three-fold, 
47 indicating that only one third of S. mutans DNA was protected within viable cells (Yasunaga et al.

48 2013). Extracellular DNA has been directly observed in mono-species $S$. mutans biofilms and is

49 actively released from cells via membrane vesicles (Liao et al. 2014). Systems for the uptake and

50 incorporation of free DNA are widely present in oral bacteria including Streptococcus spp. and

51 Porphyromonas spp., indicating that these microorganisms are exposed to eDNA at times within

52 their natural environments (Tribble et al. 2012; Jakubovics et al. 2014). Indeed, there is extensive

53 evidence from genome sequences that DNA has been horizontally transferred between oral

54 bacteria (Do et al. 2011).

55 We have recently shown that extracellular matrix material is abundant in subgingival dental

56 plaque in the form of strands and meshwork structures that appear similar to "yarn-like" threads

57 and eDNA lattice structures, or "sweaters" observed within in vitro Enterococcus faecalis biofilms

58 (Barnes et al. 2012; Holliday et al. 2015). Here, we aimed to visualize eDNA in natural oral biofilm

59 samples using specific antibodies and an ultrasensitive dye. In addition, the possible structural role

60 of eDNA was assessed in model dental plaque biofilms.

\section{MATERIALS \& METHODS}

64 Detailed methods are given in the Appendix.

\section{Clinical specimen collection}

Study participants were recruited from the Periodontology consultation clinics of the

67 Newcastle Dental Hospital. Ethical approval was obtained from the Yorkshire and Humber

68 Research Ethics Committee (Reference 14/YH/0145). Dental implants and periodontally affected

69 teeth were stored in PBS for up to $3 \mathrm{~h}$ after collection. Teeth were sectioned using a dental air 
70 turbine handpiece under water cooling. Saliva was collected as described by Nance et al. (2013).

71 In brief, parafilm-stimulated whole saliva was collected on ice from at least five healthy adults

72 who were non-smokers, had not consumed food or hot drinks for $2 \mathrm{~h}$, and had not taken antibiotics

73 for at least two weeks prior to donation. Individual saliva samples or pooled saliva was mixed with 74 glycerol in a $75 \% / 25 \%$ ratio within $2 \mathrm{~h}$ of collection and stored at $-80^{\circ} \mathrm{C}$.

75

Routine culture of bacteria and biofilm growth

E. faecalis $\mathrm{JH} 2-2$ was routinely grown in BHY (37 g/L Brain Heart Infusion and $5 \mathrm{~g} / \mathrm{L}$

78 yeast extract, Melford, Ipswich, UK). Microcosm biofilms were inoculated with human saliva,

79 diluted 1:100 in artificial saliva (Pratten 2007). In some cases, glucose in the artificial saliva was

80 replaced with $2 \%(\mathrm{w} / \mathrm{v})$ sucrose. Biofilms were cultured statically on plastic or glass surfaces (see

81 Appendix) for $24 \mathrm{~h}$ at $37^{\circ} \mathrm{C}$ either aerobically (with sucrose) or anaerobically.

83 Production and purification of NucB

Recombinant NucB from Bacillus licheniformis DSM13 was produced in Bacillus subtilis

85 NZ8900 using the SURE expression system as previously described (Nijland et al. 2010) and 86 purified as described in the Appendix.

Fluorescent staining

Biofilms were rinsed with PBS prior to fluorescent staining. To stain eDNA,

90 immunofluorescent labelling was performed as described by Barnes et al. (2012). Alternatively,

91 biofilm-harboring substrata were incubated with 2.4 nM YOYO-1 (Life Technologies, Carlsbad,

92 California, United States) for $10 \mathrm{~min}$ at $20^{\circ} \mathrm{C}$. Bacterial cell membranes were stained with $1 \mu \mathrm{g} \mathrm{ml}^{-1}$ 
93 Nile Red (Sigma-Aldrich, St. Louis, Missouri, United States) or with $2.9 \mu \mathrm{g} \mathrm{ml}^{-1}$ FM5-95 (Life

94 Technologies). Microcosm biofilms were stained with Live/Dead BacLight ${ }^{\mathrm{TM}}$ (Molecular Probes,

95 Eugene, Oregon, USA). Biofilms were visualized by confocal laser scanning microscopy (CLSM)

96 or by epifluorescent microscopy (see Appendix).

97

98 Scanning electron microscopy

99 Samples for field emission scanning electron microscopy (FESEM) imaging were fixed in

$1002 \%(\mathrm{w} / \mathrm{v})$ glutaraldehyde in Sorenson's Phosphate buffer and dehydrated through a series of 101 ethanol (Holliday et al. 2015). A FEI Helios NanoLab Dual Beam MK 2 field emission microscope 102 was used for imaging as previously described (Holliday et al. 2015).

103

104 NucB treatment of biofilms

105 To assay the inhibitory effects of NucB on biofilm growth, $5 \mu \mathrm{g} \mathrm{ml}^{-1}$ crude or purified

106 NucB was included with the growth medium during biofilm formation. Alternatively, $5 \mu \mathrm{g} \mathrm{m}{ }^{-1}$

107 NucB in $50 \mathrm{mM}$ Tris- $\mathrm{HCl}\left(\mathrm{pH} \mathrm{8.0)}\right.$ was added to biofilms after growth and incubated at $37^{\circ} \mathrm{C}$ for

$1081 \mathrm{~h}$. Biofilms were quantified by crystal violet staining (see Appendix). All assays were performed

109 in triplicate, and results shown are means and standard errors from three independent experiments.

110 Statistical significance of differences between groups was assessed by one-way ANOVA with

111 Tukey's post-hoc test.

112

\section{DNA extraction and microbial population analysis}

114 For microbial population analysis, biofilms were cultured on the surface of 6 well plates in 115 the presence or absence of purified NucB (see Appendix). DNA was extracted using the 
116 MasterPure $^{\mathrm{TM}}$ Gram Positive DNA Purification Kit (Epicentre ${ }^{\circledR}$ Biotechnologies, Madison, USA)

117 as described previously (Shields et al. 2013). Next Generation Sequencing was performed on the

118 Illumina MiSeq platform by MR DNA (Shallowater, TX, USA). See Appendix for more details. 119 


\section{RESULTS}

121 Visualization of eDNA in $E$. faecalis biofilms

Techniques for visualizing eDNA were initially developed using E. faecalis biofilms since

123 these are well-known to contain extensive eDNA (Barnes et al., 2012). Biofilms were grown on

124 glass coverslips and eDNA was visualized using fluorophore-conjugated anti-DNA antibodies.

125 Using CLSM, eDNA was concentrated in punctate foci protruding from the base of the biofilm to

126 the surface (Fig. 1A). These bright foci were consistently found throughout the biofilm suggesting

127 that eDNA was present within the matrix. There was no staining in the absence of either primary

128 or secondary antibody (data not shown). To visualize eDNA in viable (unfixed) biofilms with

129 minimal sample processing, the ultrasensitive cell-impermeant fluorescent DNA dye YOYO-1

130 was used. With this approach, eDNA appeared as web-like structures localized outside cells

131 (Fig. 1B). A few bright foci were also observed within biofilms. To obtain increased resolution,

132 biofilms were gently harvested by scraping and placed on agarose pads for visualization by 133 epifluorescent microscopy (Fig. 1C). In these images, E. faecalis cells were surrounded by eDNA.

134 YOYO-1 staining was almost always restricted to the outside of the cells, and YOYO-1 staining 135 appeared similar to that in intact biofilms (Fig. 1B). To ensure that YOYO-1 was specifically 136 staining eDNA, biofilms were treated with the DNase enzyme NucB prior to staining (Fig. 1C 137 lower panel). By quantitative image analysis, the YOYO-1 signal was significantly decreased by $138>70 \%$ after NucB treatment $(p<0.05)$.

140 Extracellular DNA in salivary microcosm models of dental plaque

141 To assess immunofluorescence and YOYO-1 staining for detection of eDNA in mixed142 species biofilms, microcosms of human salivary bacteria were cultured anaerobically in vitro on 
143 glass coverslips as a model of subgingival dental plaque growing in the low oxygen environment

144 of periodontal pockets. Once again, immunofluorescent labelling showed punctate areas of bright

145 green fluorescence, indicative of eDNA, throughout the biofilm (Fig. 2A). Staining with YOYO-1

146 also gave rise to punctate green fluorescence co-localized to areas of cells (Fig. 2B). At higher

147 resolution, the majority of the YOYO-1 stain was clearly outside cells (Fig. 2C). Similar to E.

148 faecalis biofilms, there were a few very bright regions that appeared to represent intracellular

149 YOYO-1 dye.

151 Extracellular DNA in dental plaque from ex vivo specimens

152 Samples of natural plaque from extracted teeth and implants were collected from the 153 periodontology clinics for eDNA analysis. Using FESEM, it was shown that tooth samples 154 contained extensive subgingival dental plaque (Fig. 3A). Biofilms consisted of many different 155 microbial cells with distinct morphologies surrounded by extracellular material (Fig. 3A, i). 156 Interestingly, numerous cells appeared to have holes in their walls while being held in place by 157 matrix material (Fig. 3A, ii). The matrix also included many small vesicle-like structures (Fig. 3A, 158 iii).

Direct visualization of biofilms on extracted teeth and implants was not possible due to the

160 uneven nature of the substratum and excessive autofluorescence from the substratum material.

161 Therefore, biofilms were harvested by scraping and visualized under high resolution 162 epifluorescence microscopy. Biofilms were detected on provisional resin composite crowns and 163 overdenture abutments recovered during the restorative phase of implant treatment (Fig. 3B). In 164 general, biofilms on implant crowns were more abundant and appeared to harbor more bacteria 165 than those on overdenture abutments. Nevertheless, using YOYO-1, eDNA was clearly visible in 
166 both types of biofilm. Subgingival dental plaque on the surface of teeth extracted for periodontitis

167 contained many different cell morphologies along with extracellular material that was stained with

168 YOYO-1 (Fig. 3C). Treatment with NucB reduced the extent of green staining dramatically,

169 supporting that the YOYO-1 specifically stained eDNA.

171 Importance of eDNA for biofilm structure

Initially, the activity of NucB against microcosm biofilms was assessed using crude NucB

173 preparations. Saliva from six different volunteers was used as the source of microbial inoculae and

174 biofilms were cultured either aerobically in $2 \%$ sucrose as a cariogenic biofilm model, or

175 anaerobically to mimic the low oxygen tension of the periodontal pocket environment. Inhibition

176 of biofilm formation was assessed by including NucB during biofilm growth. Alternatively,

177 dispersal of pre-formed biofilms was monitored by incubating pre-formed biofilms with NucB. No

178 aerobic, sucrose-grown biofilms were inhibited in the presence of $\mathrm{NucB}$ and only one biofilm was

179 significantly dispersed (Fig. S1). In several cases, anaerobically cultured biofilms were

180 significantly inhibited or dispersed by NucB (data not shown). To ensure that impurities in the 181 enzyme preparation were not responsible for the observed effects on anaerobic biofilms, NucB

182 was purified by sequential chromatography. Purified NucB had DNase activity but no detectable

183 RNase or protease activity (Fig. S2). Incubation with NucB significantly $(p<0.05)$ reduced biofilm

184 growth in microcosms from four distinct salivary inoculae and from pooled saliva (Fig. 4A and

185 B). Treatment of pre-formed biofilms with NucB resulted in much smaller reductions in biofilm

186 biomass that were not statistically significant (Fig. 4B).

187 To determine whether NucB selectively excluded certain micro-organisms from biofilms,

188 DNA was extracted from biofilms cultured in the presence or absence of NucB and analyzed by 
next generation sequencing. The microbial load was much lower in the presence of NucB (Fig. 5).

190 The bacterial diversity within each sample (alpha diversity) was significantly reduced in NucB-

191 treated biofilms compared with controls (Fig. S3). Control samples formed a discrete cluster by

192 principle co-ordinates analysis that was clearly separated from NucB-treated samples (Fig. S4).

193 Sixteen genera were present at $>1 \%$ of the total reads (Fig. 5A). Following NucB treatment, the

194 proportions of all genera except Actinomyces, Aggregatibacter and Fusobacterium were

195 significantly changed $(p<0.05)$. Populations cultured in the presence of NucB were enriched in

196 Streptococcus and Veillonella species, whereas Haemophilus, Neisseria, Peptostreptococcus,

197 Porphyromonas and Prevotella were dramatically reduced with NucB treatment. Haemophilus

198 parainfluenzae was the most abundant species in the absence of NucB (18\% of the population),

199 but was reduced by an order of magnitude in NucB-treated biofilms. Neisseria subflava was also

200 reduced by NucB treatment, from $11 \%$ to $1 \%$ of the total population. By contrast, Streptococcus

201 salivarius, Streptococcus parasanguinis, Veillonella parvula and Veillonella dispar were

202 proportionally increased in populations treated with NucB (Fig. 5B).

\section{DISCUSSION}

205 Exopolysaccharides in cariogenic dental plaque have been the subject of intense research 206 over many decades (Koo et al. 2013). However, the composition of matrices of non-cariogenic 207 oral biofilms is relatively poorly understood. Oral streptococci such as S. gordonii, S. sanguinis 208 and S. mutans produce eDNA in laboratory mono-cultures (Kreth et al. 2009; Liao et al. 2014). 209 Here, eDNA was also detected in mixed-species oral biofilms including subgingival dental plaque 210 and plaque on the surface of the provisional resin composite crowns and overdenture abutments of 
211 dental implants. We propose that eDNA is likely a ubiquitous component of biofilms throughout 212 the oral cavity.

213 Ideally, techniques to visualize eDNA in natural biofilms should be non-disuptive and 214 highly specific. Recently, FESEM has been applied in combination with anti-DNA antibodies or 215 DNase enzymes to show the structure of eDNA in E. faecalis or S. mutans biofilms (Barnes et al. 216 2012; Liao et al. 2014). Extracellular DNA appeared as long strands, described as 'yarn-like' 217 structures, or in weblike mats likened to 'sweaters' (Barnes et al. 2012). We have observed both 218 yarn-like and sweater structures in the matrices of subgingival dental plaque using FESEM 219 (Fig. 3A). Despite numerous attempts, we were not able to label these with specific anti-DNA 220 antibodies in conjunction with FESEM (Holliday et al. 2015). Therefore, we have developed 221 fluorescence-based methods to stain eDNA in natural biofilms. Using these techniques, eDNA was 222 detected in all biofilms that were studied here.

223 High resolution images showing eDNA surrounding cells were obtained by gently scraping 224 the biofilms and transferring them to agar pads. In optimisation experiments on model single225 species or microcosm biofilms, no qualitative differences were observed in the amount of eDNA 226 present in intact biofilms compared with harvested biofilms. In addition, the eDNA appeared 227 closely localized around cells in both intact and harvested model biofilms. Therefore, apparently 228 harvesting biofilms did not dramatically alter the nature of the eDNA. Treatment of biofilms with 229 NucB significantly reduced eDNA but did not completely eliminate it. It is possible that eDNA 230 was protected to some extent by binding to protein or carbohydrates. Previous studies have shown 231 that eDNA produced by oral bacteria including S. gordonii and Porphyromonas gingivalis 232 associates with DNA binding proteins such as DNAIIB (Rocco et al. 2016). In Staphylococcus 233 aureus, beta toxin becomes covalently linked to eDNA, forming a nucleoprotein matrix (Huseby 
234 et al. 2010). The Pseudomonas aeruginosa polysaccharide Pel cross-links eDNA by ionic 235 interactions (Jennings et al. 2015), and in the presence of neutrophils eDNA is apparently protected

236 from DNase digestion by association with F-actins or histones (Parks et al. 2009). It is possible 237 that similar mechanisms occur in oral biofilms. Alternatively, it is possible that extracellular 238 proteases in mature biofilms may have degraded NucB. Work is ongoing to identify the fate of 239 NucB following addition to biofilms.

240 In all samples studied here, there was evidence that YOYO-1 occasionally entered cells. It

241 is likely that these cells were compromised, since YOYO-1 does not cross intact membranes (Pham 242 et al. 2011). In fact, FESEM images showed that some cells in the biofilm were open at one pole, 243 and likely would not have excluded dyes. Indeed, it is possible that controlled cell lysis may be a 244 mechanism for the release of DNA into the biofilm matrix. In E. faecalis, cell lysis is actively 245 induced in a proportion of the population as a mechanism for releasing DNA into the surrounding 246 milieu (Thomas et al. 2009). More recently it has been shown that subpopulations of $P$. aeruginosa 247 undergo explosive cell lysis, releasing DNA either in free form or encapsulated within membrane 248 vesicles (Turnbull et al. 2016). Membrane vesicles were also observed in our FESEM images, and 249 these may have been the remnants of explosive lysis of cells. It is possible that eDNA may also 250 enter the biofilm without cell lysis, as has been demonstrated in E. faecalis and S. gordonii mono251 cultures (Itzek et al. 2011; Barnes et al. 2012). Neisseria gonorrhoaea releases DNA through a 252 type IV secretion system (Hamilton et al. 2005), and homologues may be present in oral bacteria. 253 We are currently developing methods to purify eDNA without lysing bacterial cells in order to 254 investigate the size and sequence of eDNA fragements within natural oral biofilms.

255 Using the DNase enzyme NucB, it was shown that eDNA plays an important structural role 256 during the formation of oral biofilms. Biofilms cultured aerobically in sucrose were relatively 
257 insensitive to NucB, presumably because extracellular polysaccharides stabilized the matrix (Koo

258 et al. 2013). Pre-formed biofilms cultured anaerobically were also not significantly reduced by

259 NucB treatment. By contrast, the formation of anaerobic biofilms was strongly inhibited in the

260 presence of NucB (Figure 4). The sensitivity of anaerobic biofilms to DNase treatment is similar

261 to that observed in mono-species $P$. aeruginosa biofilms, which are sensitive to DNase I only

262 during the early stages of biofilm formation (Whitchurch et al. 2002). It is possible that eDNA is

263 important during the initial stages of attachment and colonization. Alternatively, eDNA may

264 become resistant to DNase enzymes during biofilm maturation by forming complexes with other

265 macromolecules.

266 Interestingly, we observed species-specific effects of NucB during initial colonization of

267 oral biofilms and the microbial diversity in biofilms was significantly reduced by NucB treatment.

268 To our knowledge, this is the first demonstration that DNase treatment can cause marked shifts in

269 the microbial composition of mixed-species oral biofilms. In vitro, biofilms formed by different

270 species and even different strains within a species show marked differences in their sensitivities to

271 DNase enzymes (Lappann et al. 2010; Shields et al. 2013). It is notable that well-known pioneer

272 colonizers of dental plaque such as streptococci and veillonellae were enriched in biofilms grown

273 in the presence of NucB. Pioneer colonizers produce cell surface adhesins that recognize protein

274 or glycoprotein receptors in the salivary pellicle and mediate adhesion to tooth surfaces (Nobbs et

275 al. 2011). Therefore, these species may be less reliant on eDNA for adhesion than later colonizers.

276 By selectively targeting certain species, NucB may reduce the capacity for synergistic

277 interbacterial interactions or for the establishment of gradients of nutrients or oxygen, which are

278 essential for biofilm development and maturation. Consequently, addition of NucB during dental

279 plaque formation may have profound effects on the overall biofilm structure. 
In summary, this study provides clear evidence that eDNA is present in oral biofilms

281 including plaque on natural teeth and dental implants. Extracellular DNA represents a potential

282 target for biofilm control and for the exclusion of potential pathogens from plaque. Our data

283 indicate that DNases may be more effective for controlling biofilms in areas of low oxygen tension

284 such as subgingival dental plaque than for cariogenic supragingival plaque formed from a high-

285 sugar diet, which is characterized by an abundance of exopolysaccharides. Human DNase I is

286 already used for the treatment of cystic fibrosis, demonstrating that DNases may have potential for

287 biofilm control within the human body. Further studies are now required to determine the role of 288 eDNA in the struture and stability of mature oral biofilms, and to assess the potential for DNase 289 enzymes to be used in oral healthcare applications.

\section{ACKNOWLEDGEMENTS}

292 We are grateful for the technical support of Trevor Booth in the Bioimaging Facility and

293 Tracey Davey at the Electron Microscopy Research Services, Newcastle University. We gratefully

294 acknowledge the expert technical assistance of Lesley Old, Newcastle University. We thank Aaron

295 Barnes and Gary Dunny (University of Minnesota) for helpful discussions and Waleed

296 Mohammed, Newcastle University, for assistance with conducting important control experiments.

297 We are grateful to the Centre for Oral Health Research, Newcastle University, for supporting NR

298 with a Bradlaw Postdoctoral Fellowship. There are no conflicts of interest in relation to this work. 299

\section{$300 \quad$ FUNDING}

301 This research received no specific grant from any funding agency in the public, 302 commercial, or not-for-profit sectors. 


\section{REFERENCES}

304 Barnes AM, Ballering KS, Leibman RS, Wells CL, Dunny GM. 2012. Enterococcus faecalis produces abundant extracellular structures containing DNA in the absence of cell lysis during early biofilm formation. MBio 3(4):e00193-00112.

Chen T, Yu WH, Izard J, Baranova OV, Lakshmanan A, Dewhirst FE. 2010. The Human Oral Microbiome Database: a web accessible resource for investigating oral microbe taxonomic and genomic information. Database (Oxford) 2010:baq013.

Do T, Gilbert SC, Klein J, Warren S, Wade WG, Beighton D. 2011. Clonal structure of Streptococcus sanguinis strains isolated from endocarditis cases and the oral cavity. Mol Oral Microbiol 26(5):291-302.

Dominiak DM, Nielsen JL, Nielsen PH. 2011. Extracellular DNA is abundant and important for microcolony strength in mixed microbial biofilms. Environ Microbiol 13(3):710-721.

Hamilton HL, Dominguez NM, Schwartz KJ, Hackett KT, Dillard JP. 2005. Neisseria gonorrhoeae secretes chromosomal DNA via a novel type IV secretion system. Mol Microbiol 55(6):1704-1721.

Holliday R, Preshaw PM, Bowen L, Jakubovics NS. 2015. The ultrastructure of subgingival dental plaque, revealed by high-resolution field emission scanning electron microscopy. Bdj Open $1: 15003$.

Huseby MJ, Kruse AC, Digre J, Kohler PL, Vocke JA, Mann EE, Bayles KW, Bohach GA, Schlievert PM, Ohlendorf DH, et al. 2010. Beta toxin catalyzes formation of nucleoprotein matrix in staphylococcal biofilms. Proc Natl Acad Sci U S A 107(32):14407-14412.

Itzek A, Zheng L, Chen Z, Merritt J, Kreth J. 2011. Hydrogen peroxide-dependent DNA release and transfer of antibiotic resistance genes in Streptococcus gordonii. J Bacteriol 193(24):6912-6922.

Jakubovics NS, Shields RC, Rajarajan N, Burgess JG. 2013. Life after death: the critical role of extracellular DNA in microbial biofilms. Lett Appl Microbiol 57(6):467-475.

Jakubovics NS, Yassin SA, Rickard AH. 2014. Community interactions of oral streptococci. Adv Appl Microbiol 87:43-110.

Jennings LK, Storek KM, Ledvina HE, Coulon C, Marmont LS, Sadovskaya I, Secor PR, Tseng BS, Scian M, Filloux A, et al. 2015. Pel is a cationic exopolysaccharide that cross-links extracellular DNA in the Pseudomonas aeruginosa biofilm matrix. Proc Natl Acad Sci U S A 112(36):11353-11358.

Koo H, Falsetta ML, Klein MI. 2013. The exopolysaccharide matrix: a virulence determinant of cariogenic biofilm. J Dent Res 92(12):1065-1073.

Kreth J, Vu H, Zhang Y, Herzberg MC. 2009. Characterization of hydrogen peroxide-induced DNA release by Streptococcus sanguinis and Streptococcus gordonii. J Bacteriol 191(20):6281-6291.

Lappann M, Claus H, van Alen T, Harmsen M, Elias J, Molin S, Vogel U. 2010. A dual role of extracellular DNA during biofilm formation of Neisseria meningitidis. Mol Microbiol 75(6):1355-1371.

Liao S, Klein MI, Heim KP, Fan Y, Bitoun JP, Ahn SJ, Burne RA, Koo H, Brady LJ, Wen ZT. 2014. Streptococcus mutans extracellular DNA is upregulated during growth in biofilms, actively released via membrane vesicles, and influenced by components of the protein secretion machinery. J Bacteriol 196(13):2355-2366. 
Nance WC, Dowd SE, Samarian D, Chludzinski J, Delli J, Battista J, Rickard AH. 2013. A highthroughput microfluidic dental plaque biofilm system to visualize and quantify the effect of antimicrobials. J Antimicrob Chemother 68(11):2550-2560.

Nijland R, Hall MJ, Burgess JG. 2010. Dispersal of biofilms by secreted, matrix degrading, bacterial DNase. PLoS ONE 5(12):e15668.

Nobbs AH, Jenkinson HF, Jakubovics NS. 2011. Stick to your gums: mechanisms of oral microbial adherence. J Dent Res 90(11):1271-1278.

Okshevsky M, Regina VR, Meyer RL. 2014. Extracellular DNA as a target for biofilm control. Curr Opin Biotechnol 33C:73-80.

Okshevsky M, Meyer RL. 2015. The role of extracellular DNA in the establishment, maintenance and perpetuation of bacterial biofilms. Crit Rev Microbiol 41(3):341-352.

Otsuka R, Imai S, Murata T, Nomura Y, Okamoto M, Tsumori H, Kakuta E, Hanada N, Momoi Y. 2015. Application of chimeric glucanase comprising mutanase and dextranase for prevention of dental biofilm formation. Microbiol Immunol 59(1):28-36.

Parks QM, Young RL, Poch KR, Malcolm KC, Vasil ML, Nick JA. 2009. Neutrophil enhancement of Pseudomonas aeruginosa biofilm development: human F-actin and DNA as targets for therapy. J Med Microbiol 58(Pt 4):492-502.

Pham TA, Kawai S, Kono E, Murata K. 2011. The role of cell wall revealed by the visualization of Saccharomyces cerevisiae transformation. Curr Microbiol 62(3):956-961.

Pratten J. 2007. Growing oral biofilms in a constant depth film fermentor (CDFF). Curr Protoc Microbiol Chapter 1:Unit 1B 5.

Ragunath C, DiFranco K, Shanmugam M, Gopal P, Vyas V, Fine DH, Cugini C, Ramasubbu N. 2016. Surface display of Aggregatibacter actinomycetemcomitans autotransporter Aae and dispersin B hybrid act as antibiofilm agents. Mol Oral Microbiol 31(4):329-339.

Rajarajan N, Ward AC, Burgess JG, Glassey J. 2012. Use of physiological information and process optimisation enhances production of extracellular nuclease by a marine strain of Bacillus licheniformis. Bioresour Technol 130C:552-558.

Rocco CJ, Davey ME, Bakaletz LO, Goodman SD. 2016. Natural antigenic differences in the functionally equivalent extracellular DNABII proteins of bacterial biofilms provide a means for targeted biofilm therapeutics. Mol Oral Microbiol:In press, doi: 10.1111/omi.12157.

Shields RC, Mokhtar N, Ford M, Hall MJ, Burgess JG, ElBadawey MR, Jakubovics NS. 2013. Efficacy of a marine bacterial nuclease against biofilm forming microorganisms isolated from chronic rhinosinusitis. PLoS ONE 8(2):e55339.

Thomas VC, Hiromasa Y, Harms N, Thurlow L, Tomich J, Hancock LE. 2009. A fratricidal mechanism is responsible for eDNA release and contributes to biofilm development of Enterococcus faecalis. Mol Microbiol 72(4):1022-1036.

Tribble GD, Rigney TW, Dao DH, Wong CT, Kerr JE, Taylor BE, Pacha S, Kaplan HB. 2012. Natural competence is a major mechanism for horizontal DNA transfer in the oral pathogen Porphyromonas gingivalis. MBio 3(1):e00231-00211

Turnbull L, Toyofuku M, Hynen AL, Kurosawa M, Pessi G, Petty NK, Osvath SR, CarcamoOyarce G, Gloag ES, Shimoni R, et al. 2016. Explosive cell lysis as a mechanism for the biogenesis of bacterial membrane vesicles and biofilms. Nat Commun 7:11220.

Whitchurch CB, Tolker-Nielsen T, Ragas PC, Mattick JS. 2002. Extracellular DNA required for bacterial biofilm formation. Science 295(5559):1487. 
Yasunaga A, Yoshida A, Morikawa K, Maki K, Nakamura S, Soh I, Awano S, Ansai T. 2013. Monitoring the prevalence of viable and dead cariogenic bacteria in oral specimens and in vitro biofilms by qPCR combined with propidium monoazide. BMC Microbiol 13:157.

Zhang M, Chen Y, Xie L, Li Y, Jiang H, Du M. 2015. Pyrosequencing of Plaque Microflora In Twin Children with Discordant Caries Phenotypes. PLoS One 10(11):e0141310. 


\section{FIGURE LEGENDS}

Figure 1: Visualization of eDNA in E. faecalis biofilms. (A) Immunofluorescent labelling of $E$. faecalis biofilm with an $\alpha$-DNA antibody (green). Nile Red was included to highlight cell membrane (red). A 3D rendering of the overlay image is shown on the left. The right panel shows Alexa flour-antibody labelled eDNA forming punctate foci protruding from the base of the biofilm. (B) Fluorescent staining of E. faecalis biofilm with YOYO-1. A 3D rendering of the overlay images of YOYO-1 stained eDNA (green) and Nile Red stained membrane (red) is shown in left panel; YOYO-1 staining of eDNA within the biofilm is shown on the right. Dashed square highlights a region with eDNA forming web-like structures mainly localised outside the cells. An enlarged image of this region is shown in lower panel as a 3D rendering of overlay (left), eDNA

stained YOYO-1 (centre) and overlay image (right). The arrow indicates two cells apparently permeable to YOYO-1 dye. (C) NucB treatment of E. faecalis biofilm leads to decreased eDNA signal in abraded cells. Epifluorescent images of abraded cells of E. faecalis biofilm stained with YOYO-1 (green) and FM-595 (red).

Figure 2: Detection of eDNA in model dental plaque. (A) Immunofluorescent labelling of eDNA in model plaque with $\alpha$-DNA antibody (green) and Nile Red membrane dye (red). (B) Fluorescent staining of eDNA with YOYO-1 (green) and Nile Red (red). (C) eDNA visualized in cells harvested from model dental plaque using YOYO-1 (green) and FM5-95 (red). Cells that appear permeable to YOYO-1 are highlighted with an asterisk. 
Figure 3: Identification of eDNA within natural plaque found on the surface of ex vivo specimens. (A) FESEM images of dental plaque found on the surface of a tooth extracted due to periodontitis showing a multispecies microbial community. i) Mesh-like structures of extracellular material are apparent (arrow). ii) Ruptured cells surrounded by matrix material. Asterisks highlight cells that appear to have a hole in their wall. iii) Vesicles appear on the surface of many cells throughout the biofilm (dashed square). (B) Visualization of eDNA with YOYO-1 (green) in cells harvested from supragingival (provisional resin composite crowns; top panel) or subgingival (overdenture abutments of dental implants; lower panel) regions of dental implants recovered during the restorative phase of implant treatment. FM5-95 membrane stain was utilised to highlight cell boundaries (red). (C) YOYO-1 and FM5-95 stained subgingival dental plaque isolated from the surface of an extracted tooth from a periodontal patient shows presence of eDNA. Treatment of the dental plaque with NucB prior to staining decreased the eDNA signal (green).

Figure 4: Impact of NucB on salivary microcosm biofilms. Biofilms were cultured on the surface of glass slides (A) or microtitre plates (B). (A) Biofilms cultured in the absence or presence of NucB were stained with BacLight LIVE:DEAD stain and visualized by CLSM. 3D renderings (left) or maximum projection images (right) are shown. Live cells stained with Syto 9 appear green; red staining shows eDNA or compromised cells that have taken up propidium iodide. Images were quantified using COMSTAT and the mean $+/$ - standard errors of biovolume or average thickness from three independent controls and NucB-treated samples are shown in the table. The total biovolume was significantly lower in NucB-treated samples than control $(p<0.05$, indicated by an asterisk). (B) Biofilms formed from the microorganisms present in four different individuals (1-4) or in pooled saliva were quantified by staining with crystal violet. Dark bars show controls without 
NucB treatment, white bars represent biofilm cultured in the presence or NucB (inhibition assays) or biofilms treated with NucB after culturing (dispersal). Bars represent means from three independent experiments and standard errors are shown.

Figure 5: Microbial population shifts caused by the presence of NucB during growth. (A) Sixteen genera were present at $>1 \%$ of the population in control (untreated) samples. Treatment with NucB reduced the total microbial population and shifted the composition of different genera. To facilitate comparison with the control, normalised data are shown on the right. These were obtained by multiplying all 'NucB-treated' values by a constant so that the total number of reads was the same as the control. (B) Proportions of the major species present in control samples (red circles) or NucB-treated samples (blue circles). Circles represent mean values from three independent experiments and 95\% CIs are shown. Red asterisks indicate species that were present in significantly different proportions following NucB treatment compared with controls. 


\section{Appendix: A Critical Role for Extracellular DNA in Dental Plaque}

\section{Formation}

Nadia Rostami, Robert C. Shields, Sufian A. Yassin, Alastair R. Hawkins, Leon Bowen, Ting L. Luo, Alexander H. Rickard, Richard Holliday, Philip M. Preshaw and Nicholas S. Jakubovics*

*Corresponding author

\section{List of contents:}

\section{Supplementary Methods}

1. Biofilm culture and crystal violet staining.

2. Fluorescent staining and microscopy.

3. Purification and analysis of recombinant NucB.

4. Next generation sequencing and data analysis.

\section{Supplementary Figures}

Figure S1: Effects of NucB on saliva microcosm biofilms formed aerobically in the presence of sucrose.

Figure S2: NucB purification and enzyme activity.

Figure S3: Alpha diversity in microcosm biofilms treated with PBS (control) or NucB.

Figure S4: Between-sample (beta) diversity of the six individual microcosm biofilms, estimated by PCoA using Bray-Curtis distances.

\section{Supplementary References}




\section{Supplementary Methods}

\section{Biofilm culture and crystal violet staining}

Biofilms for crystal violet assays or for microbial population analysis were cultured directly on the surface of Cellstar ${ }^{\circledR}$ 96-well microtiter plates or 6-well tissue culture plates (Greiner Bio-one, Stonehouse, UK). Alternatively, for experiments involving microscopy, biofilms were grown on sterile $13 \mathrm{~mm}$ diameter glass coverslips. Biofilms were cultured for $24 \mathrm{~h}$ at $37^{\circ} \mathrm{C}$ aerobically or anaerobically, in a $90 \% \mathrm{~N}_{2} / 5 \% \mathrm{H}_{2} / 5 \% \mathrm{CO}_{2}$ environment (Bug Box Plus, Baker Ruskinn, Sanford, Maine, USA). For quantification of biomass, biofilms were submerged in $100 \mu \mathrm{L}$ of $0.5 \%$ (w/v) crystal violet. After incubation at $20^{\circ} \mathrm{C}$ for $15 \mathrm{~min}$, wells were washed three times in $200 \mu \mathrm{L}$ of PBS, air-dried, and residual crystal violet was dissolved with $100 \mu \mathrm{L}$ of $7 \%$ (v/v) acetic acid and quantified by measuring $A 570$.

For microbial population analysis, biofilms were cultured for $24 \mathrm{~h}$ at $37^{\circ} \mathrm{C}$ in the presence or absence of purified NucB. Medium was discarded and biofilms were harvested in $1 \mathrm{ml}$ PBS using a tissue culture scraper. Cells were pelleted by centrifugation for $10 \mathrm{~min}$ at $4{ }^{\circ} \mathrm{C}$ and $3,800 \mathrm{~g}$. DNA was extracted by incubation in $150 \mu \mathrm{l}$ spheroplasting buffer containing $37.5 \mu \mathrm{g}$ lysozyme and $50 \mathrm{U}$ mutanolysin (Sigma-Aldrich), and purified with the MasterPure ${ }^{\mathrm{TM}}$ Gram Positive DNA Purification Kit (Epicentre® Biotechnologies, Madison, USA).

\section{Fluorescent staining and microscopy}

Immunofluorescent labelling of eDNA was performed as described by Barnes et al. (2012). Briefly, samples were blocked with $2 \%$ bovine serum albumin in Hanks' Buffered Saline Solution (HBSS) and labelled with a mouse anti-dsDNA monoclonal antibody (Abcam). After primary labelling and repeated washings with HBSS containing $0.5 \%$ bovine serum albumin, Alexa Fluor 
488-conjugated secondary antibody was added. Alternatively, eDNA was stained with the cellimpermeant fluorescent dye YOYO-1. Biofilm-harboring substrata were incubated with $2.4 \mathrm{nM}$ YOYO-1 (Life Technologies) for $10 \mathrm{~min}$ at $20^{\circ} \mathrm{C}$. To visualize cell membranes in intact biofilms, the biofilm-harboring substrata were incubated with $1 \mu \mathrm{g} \mathrm{ml}^{-1}$ Nile Red (Sigma-Aldrich) for 30 $\min$ at $20^{\circ} \mathrm{C}$ prior to visualization. Alternatively, to visualize the cell membrane of cells removed from the biofilm, cells were spotted on a $1.5 \%\left(\mathrm{w} / \mathrm{v}\right.$ ) agarose pad containing $2.9 \mu \mathrm{g} \mathrm{ml}^{-1}$ FM5-95 (Life Technologies). Live and dead cells within microcosm biofilms were stained with Live/Dead Bac Light ${ }^{\mathrm{TM}}$ stain (Molecular Probes).

For confocal laser scanning microscopy (CLSM), labelled or stained coverslips were rinsed with PBS and inverted onto a PBS filled rubber frame secured on a microscope slide. Imaging was performed using a Nikon A1R confocal laser scanning microscope fitted with CFI PLAN APO VC objective (Nikon 60x/1.40 Oil). Images were captured with NIS-Elements C (v4.4, Nikon) software and processed using Imaris (v8.2, Bitplane) software. Epifluorescent microscopy was performed on an inverted epifluorescence microscope (Zeiss Axiovert 200M) fitted with a PlanNeofluor objective (Zeiss 100×/1.30 Oil Ph3). A GFP filter (460/500 nm excitation 510/560 nm emission) was employed for visualization of YOYO-1 and a TRITC filter (excitation 528/556 nm; emission 571/616 nm) was used for FM5-95. Digital images were acquired and analyzed using METAMORPH software (V.6.2r6, Molecular Devices, Sunnyvale, CA, USA) or other programs as described below.

To measure fluorescence intensity of eDNA, ImageJ software (Schneider et al. 2012) was used as described by McCloy et al. (2014) and modified to fit the purposes of this work. In brief, an entire image was selected using the box tool. Manually determined thresholding was applied to remove very bright foci resulting from intracellular accumulation of dye. The mean fluorescence 
(mean gray value) was measured for 8 independent fields of view for each sample. In a similar manner, background reading was determined from dark areas within several different fields and averaged. The average background reading was subtracted from fluorescence intensity readings obtained from each image. The statistical significance of differences between fluorescence intensities of untreated and NucB-treated samples was determined using Student's t test.

For quantification of biofilms in CSLM images, the images were first rendered in Imaris software (Bitplane, Zurich, Switzerland). Image stacks were imported into ImageJ (Schneider et al. 2012) and analyzed using the COMSTAT2 plugin (Heydorn et al. 2000). Three independent samples were quantified for each condition (untreated and NucB-treated). Differences between untreated and NucB-treated samples were assessed for significance using Student's t test.

\section{Purification and analysis of recombinant NucB.}

NucB was expressed in a B. subtilis expression system as described in the Methods. Crude NucB was used as described by Shields et al. (2013). Alternatively, secreted NucB secreted was precipitated from cell-free spent growth medium by making it $65 \%$ saturated with ammonium sulphate and incubating for $15 \mathrm{~h}$ at $60^{\circ} \mathrm{C}$. NucB was recovered as a pellet by centrifugation at $10,000 \mathrm{~g}$ for $60 \mathrm{~min}$ at $40^{\circ} \mathrm{C}$. The NucB-containing pellet was dissolved in minimum volume of $50 \mathrm{mM}$ potassium phosphate $\mathrm{pH}$ 7.2, $1 \mathrm{mM}$ dithiothreitol (buffer 1). All subsequent chromatography steps were performed at $60^{\circ} \mathrm{C}$. Following clarification by centrifugation at 10,000 $g$ for $60 \mathrm{~min}$ at $40^{\circ} \mathrm{C}$, the soluble protein was dialyzed against buffer 1 at $60^{\circ} \mathrm{C}$ and loaded onto a Q sepharose column previously equilibrated with buffer 1. Following a wash with buffer 1, the sample flow through and column wash were collected as individual fractions and assayed by SDS

PAGE (12\% separating gel) for the presence of NucB. NucB-containing fractions were pooled 
appropriately and loaded onto a hydroxyapatite column previously equilibrated with buffer 1 . The column was washed with buffer 1 and the sample flow through and column wash were collected as individual fractions. Following assay of individual fractions by SDS PAGE (12\% separating gel) and UV absorbance spectrum, NucB-containing fractions were pooled appropriately. Using this procedure $50 \mathrm{mg}$ of $\mathrm{NucB}$ at $>95 \%$ purity was routinely recovered from a starting cell culture volume of $10 \mathrm{~L}$.

The purity of NucB was assessed using multiple independent and complementary methods. The presence of any nucleic acid contamination was assessed by measuring the UV absorption spectrum from $400-240 \mathrm{~nm}$. Preparations had a typical $A_{260} / A_{280}$ ratio of 0.57 (+/- 0.2$)$ indicating an absence of nucleic acid contamination. Purity in terms of protein content was assessed by overloading an SDS PAGE gel (Fig. S2A) and by differential scanning calorimetry analysis, which showed a single well defined thermal unfolding transition with a typical $\mathrm{Tm}$ in Tris- $\mathrm{HCl} \mathrm{pH} 8.0$ of $57.1^{\circ} \mathrm{C}\left(+/-0.3^{\circ} \mathrm{C}\right)$. To ensure that the single band seen in overloaded SDS PAGE analysis consisted only of NucB, bands were cut from acrylamide gels, the protein was eluted from the gel slices and subject to peptide mass fingerprinting after digestion with trypsin. Analysis carried out using the MASCOT program showed that only a single protein was present that had a significant match in the protein database. This protein was NucB and had a protein score of 152 . The protein score is $-10^{*} \log (\mathrm{P})$, where $\mathrm{P}$ is the probability that the observed match is a random event and, in this experiment, protein scores greater than 83 are significant $(p<0.05)$. A further indication of purity of NucB was provided by the observation that purified NucB crystallized under defined conditions thereby facilitating determination of its structure (manuscript in preparation).

\section{Next generation sequencing and data analysis.}


For next generation DNA sequencing on the Illumina MiSeq platform, the 16S rRNA gene V4 variable region was amplified using PCR primers 515/806 with a barcode on the forward primer. Up to 100 samples were pooled, purified and used to prepare a DNA library. Sequences obtained were initially processed using a custom MR DNA analysis pipeline (MR DNA, Shallowater, TX, USA). Sequences were joined and depleted of barcodes. Sequences $<150 \mathrm{bp}$ and sequences with ambiguous base calls were removed. Data were denoised, operational taxonomic units (OTUs) were defined by clustering at 3\% divergence (97\% similarity) and chimeras were removed. Final OTUs were taxonomically classified using BLASTn against a curated database derived from NCBI, RDPII and GreenGenes (DeSantis et al. 2006). Differences between the proportions of genera and species in biofilms following NucB treatment compared with controls were assessed for statistical significance using T-tests with a Benjamini-Hochberg false discovery rate (FDR) correction set at $\alpha=0.05$ (Reiner et al. 2003).

Alpha diversity was estimated using the Shannon-Weaver index. Shannon-Weaver was calculated after randomly selecting 10,000 reads for each sample. This process was bootstrapped over 100 iterations with read replacement. The average Shannon-Weaver score for each sample was calculated and grouped by presence or absence of NucB. A Kruskal-Wallis non-parametric test was performed to determine differences in Shannon-Weaver score between NucB-treated biofilms and biofilms developed in the absence of NucB. Significance threshold was set at $\alpha=0.05$. Beta diversity was visualized on a principle coordinates analysis (PCoA) ordination plot using Bray-Curtis distances. Samples were rarefied to even depth according to the sample with the lowest read count. The OTU table was then fed as input in Phyloseq's ordinate function in R in order to reduce dimensionality down to 2 , where the $\mathrm{x}$-axis explains the most variation attributable to community taxa distribution by sample, and the y-axis explains the second most variation. A visual 
determination was used to conclude whether biofilms treated with NucB can be distinguished from biofilms not treated with NucB. Raw data from Illumina sequencing are stored on the Illumina BaseSpace platform and can be obtained by contacting the corresponding author. 


\section{Supplementary Figure 1}
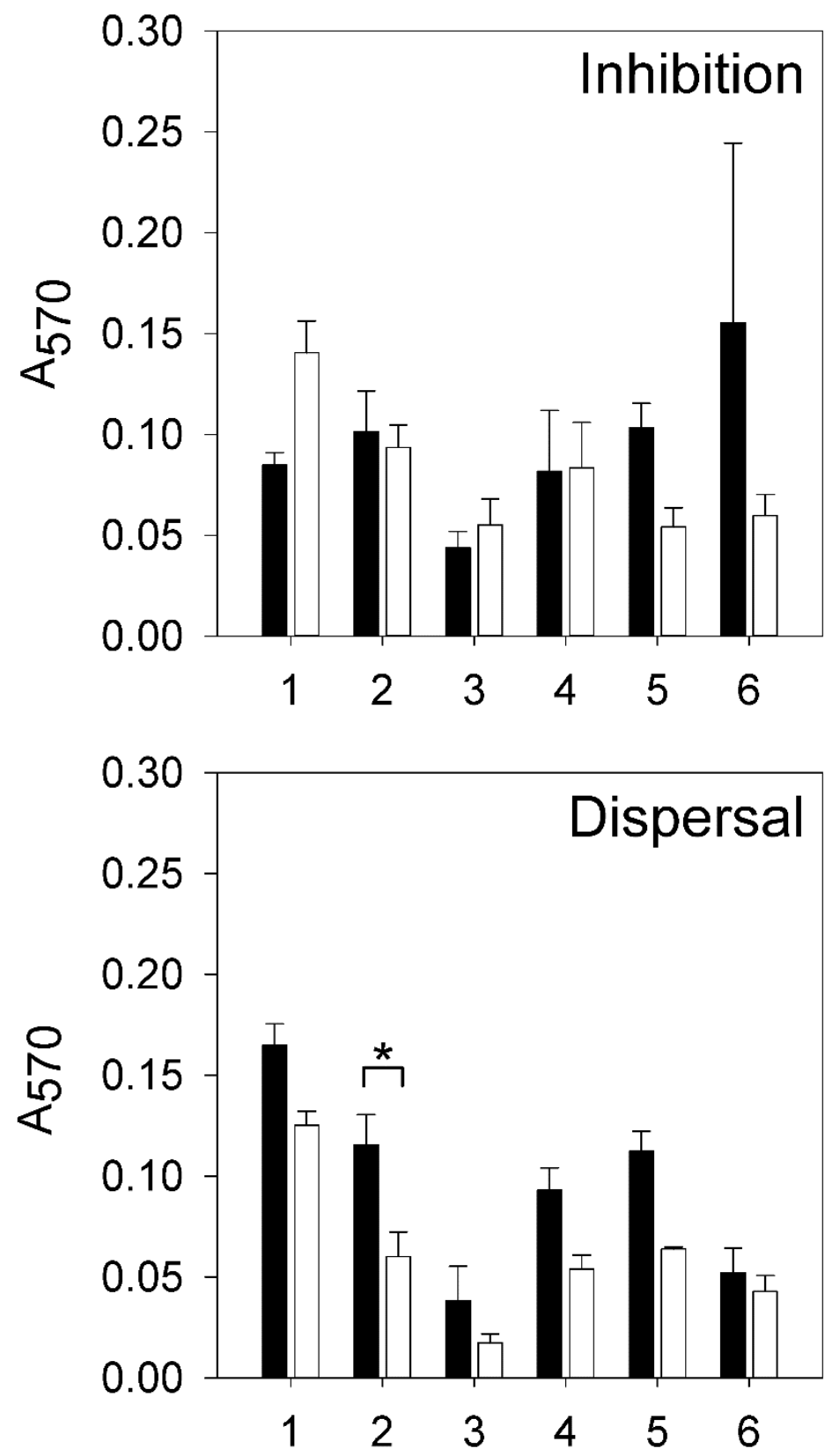

Figure S1. Effects of NucB on saliva microcosm biofilms formed aerobically in the presence of sucrose. Biofilms were cultured from salivary inocula of 6 different volunteers in the presence of NucB ('inhibition'; upper panel) or pre-formed biofilms were incubated with NucB ('dispersal'; lower panel). Biofilms were quantified by staining with crystal violet and reading $A_{570}$. Mean and standard error of control samples (dark bars) or NucB-treated samples (white bars) are shown. Significant difference $(p<0.05)$ between NucB treated sample and matched control is indicated by an asterisk. 


\section{Supplementary Figure 2}
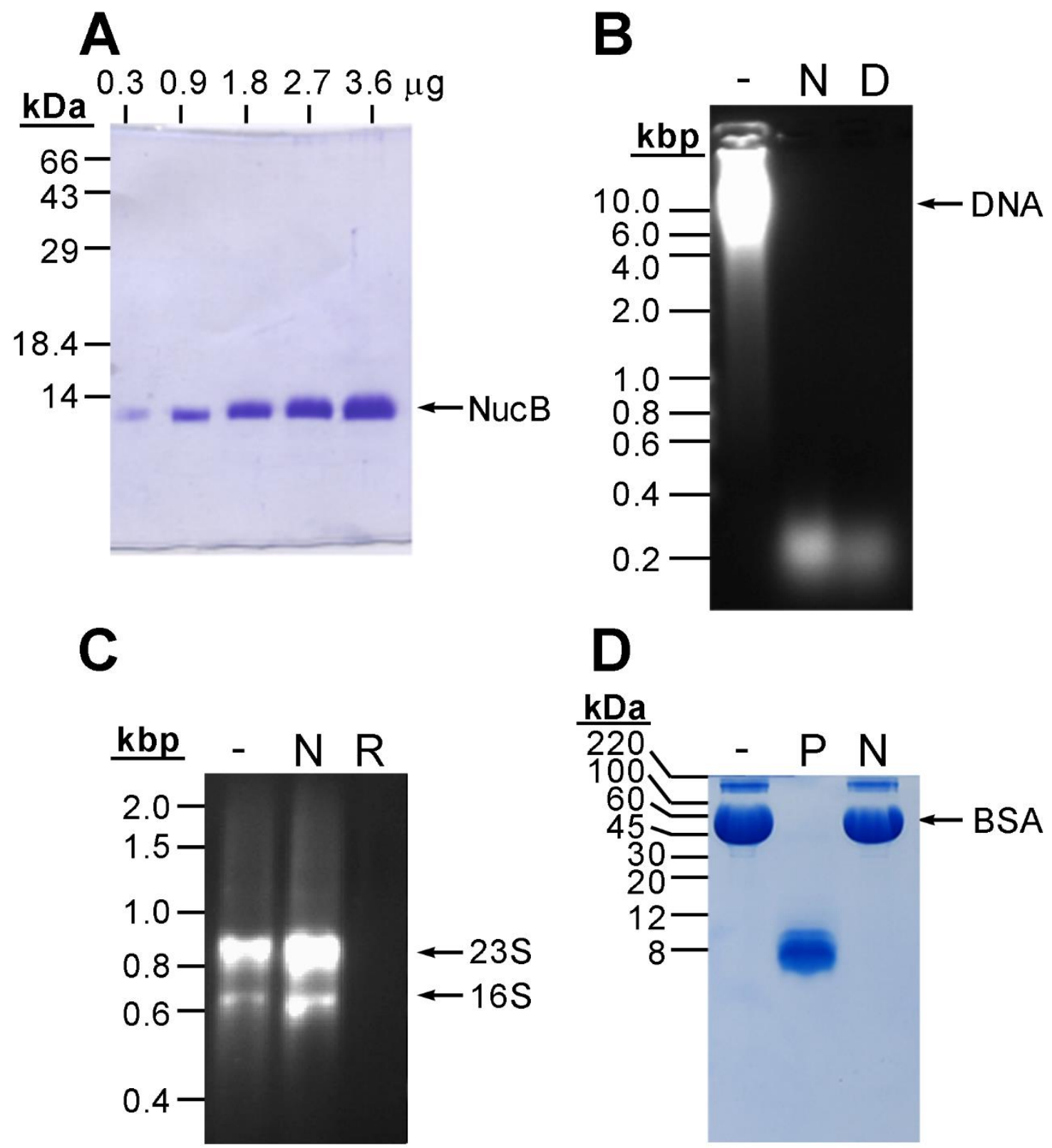
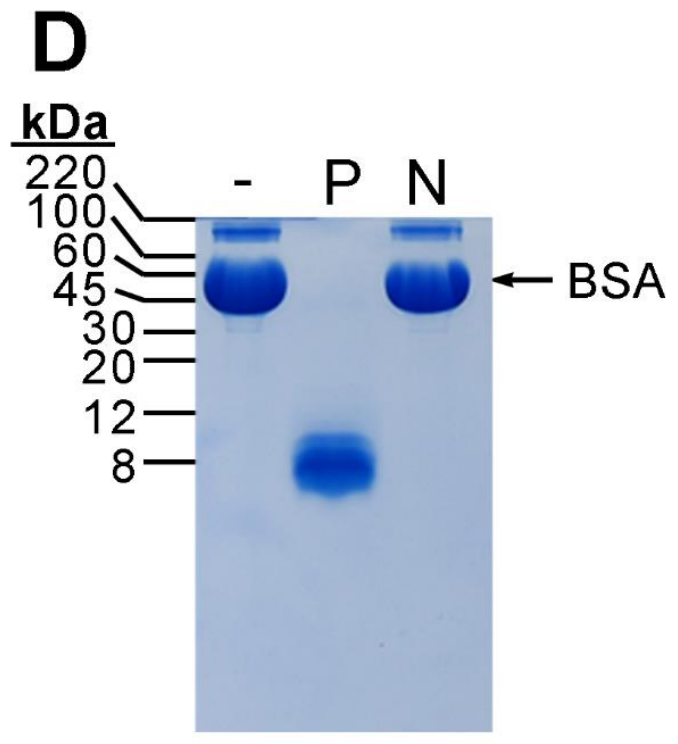

Figure S2. NucB purification and enzyme activity. The purity of $\mathrm{NucB}(\sim 12 \mathrm{kDa})$ preparations was assessed by SDS PAGE and staining with Coomassie Brilliant Blue, loaded with different amounts of protein (A). B: DNase activity of $\mathrm{NucB}(\mathrm{N})$ against calf thymus DNA was compared with a negative control (-) and DNase I (labelled 'D'). C: RNase activity of NucB against purified total bacterial RNA from Veillonella parvula, compared with RNase A (R). The 23S and 16S rRNA bands are indicated. D: Protease activity of NucB against bovine serum albumin compared with Proteinase K (P). NucB preparations had DNase activity but no detectable RNase or protease. 
Supplementary Figure 3

\section{Shannon Diversity of NucB and PBS Treated Samples}

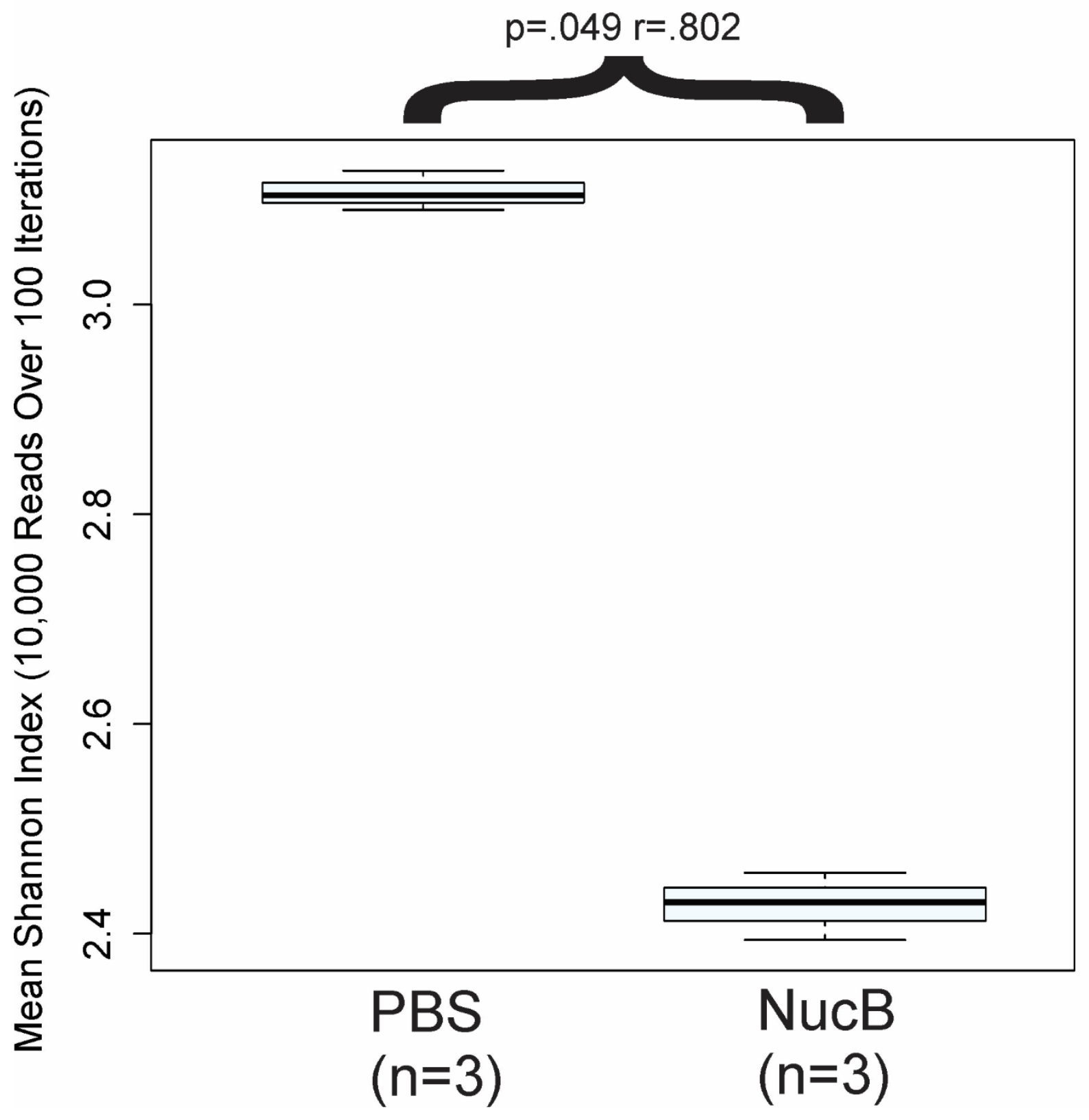

Figure S3. Alpha diversity in microcosm biofilms treated with PBS (control) or NucB. The Shannon Weaver Index was calculated as described in the Methods. The alpha diversity of biofilms treated with NucB was significantly lower than controls. 


\section{Supplementary Figure 4}

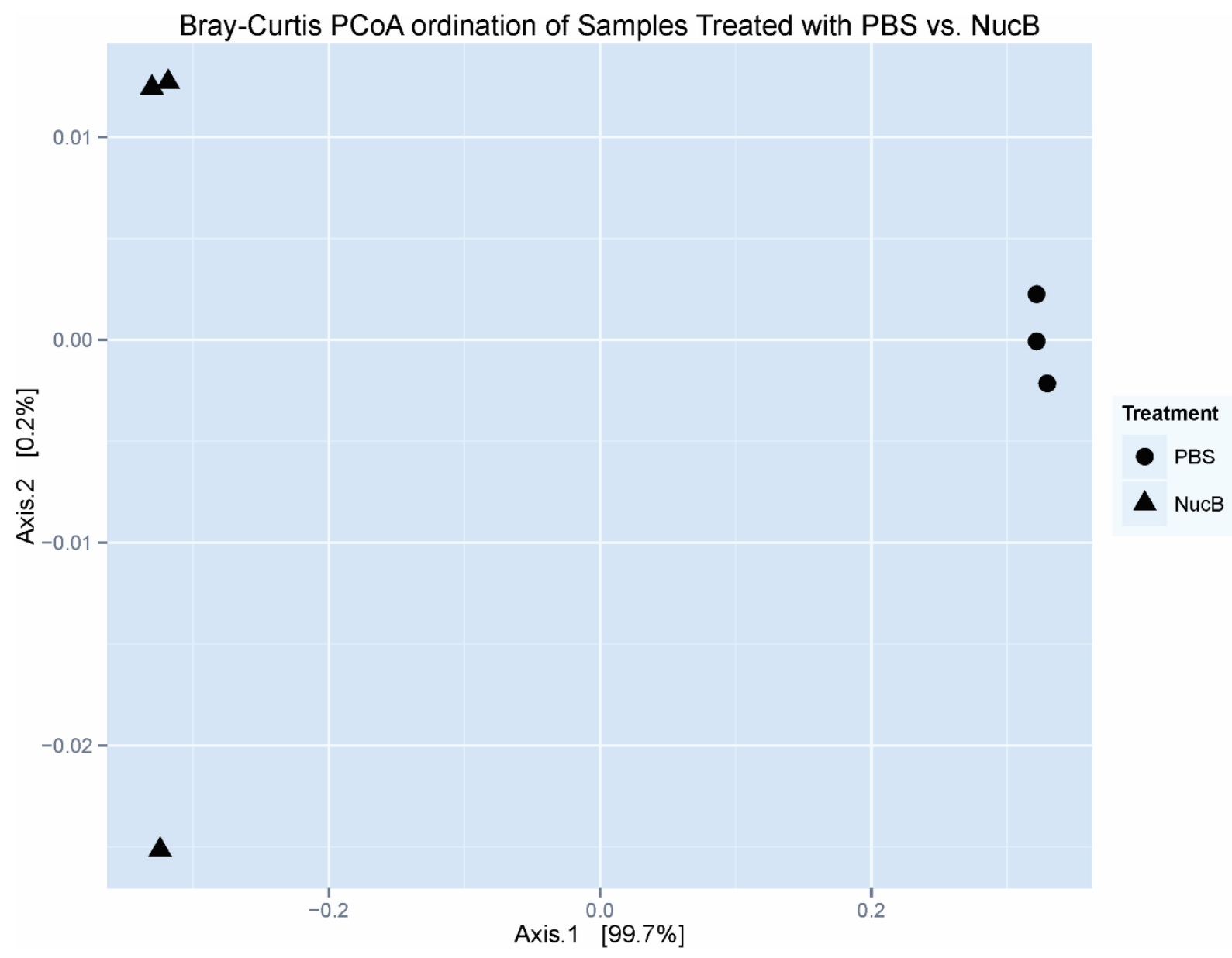

Figure S4. Between-sample (beta) diversity of the six individual microcosm biofilms, estimated by PCoA using Bray-Curtis distances. Control (PBS-treated) microcosm biofilms clustered together and were well-separated from NucB-treated biofilm samples. 


\section{Supplementary References}

Barnes AM, Ballering KS, Leibman RS, Wells CL, Dunny GM. 2012. Enterococcus faecalis produces abundant extracellular structures containing DNA in the absence of cell lysis during early biofilm formation. MBio 3(4):e00193-00112.

DeSantis TZ, Hugenholtz P, Larsen N, Rojas M, Brodie EL, Keller K, Huber T, Dalevi D, Hu P, Andersen GL. 2006. Greengenes, a chimera-checked 16S rRNA gene database and workbench compatible with ARB. Appl Environ Microbiol 72(7):5069-5072.

Heydorn A, Nielsen AT, Hentzer M, Sternberg C, Givskov M, Ersboll BK, Molin S. 2000. Quantification of biofilm structures by the novel computer program COMSTAT. Microbiology 146(10):2395-2407.

McCloy RA, Rogers S, Caldon CE, Lorca T, Castro A, Burgess A. 2014. Partial inhibition of Cdk1 in G 2 phase overrides the SAC and decouples mitotic events. Cell Cycle 13(9):1400-1412.

Reiner A, Yekutieli D, Benjamini Y. 2003. Identifying differentially expressed genes using false discovery rate controlling procedures. Bioinformatics 19(3):368-375.

Schneider CA, Rasband WS, Eliceiri KW. 2012. NIH Image to ImageJ: 25 years of image analysis. Nat Methods 9(7):671-675.

Shields RC, Mokhtar N, Ford M, Hall MJ, Burgess JG, ElBadawey MR, Jakubovics NS. 2013. Efficacy of a marine bacterial nuclease against biofilm forming microorganisms isolated from chronic rhinosinusitis. PLoS ONE 8(2):e55339. 